\section{Kidney Blood Pressure Research}

Kidney Blood Press Res 2018;43:970-978

DOI: $10.1159 / 000490686$

Published onlıne: 18 June, 2018

Accepted: 7 June, 2018

This article is licensed under the Creative Commons Attribution-NonCommercial-NoDerivatives 4.0 InternaThis article is licensed under the Creative Commons Attribution-NonCommercial-NoDerivatives 4.0 Interna-
tional License (CC BY-NC-ND) (http://www.karger.com/Services/OpenAccessLicense). Usage and distribution for commercial purposes as well as any distribution of modified material requires written permission.

\title{
Progression of Chronic Kidney Disease Affects HDL Impact on Lipoprotein Lipase (LPL)-Mediated VLDL Lipolysis Efficiency
}

\author{
Agnieszka Ćwiklińska ${ }^{a}$ Monika Cackowskab Ewa Wieczorek $^{\mathrm{a}}$ Ewa Król ${ }^{\mathrm{b}}$ \\ Robert Kowalskic Agnieszka Kuchta ${ }^{a}$ Barbara Kortas-Stempak ${ }^{a}$ Anna Gliwińska \\ Kamil Dąbkowskia Justyna Zielińska ${ }^{a} \quad$ Alicja Dębska-Ślizieńb Maciej Jankowskia \\ aDepartment of Clinical Chemistry, Medical University of Gdańsk, Gdańsk, bDepartment of Nephrology, \\ Transplantology and Internal Diseases, Medical University of Gdańsk, Gdańsk, 'Department of \\ Therapeutic Drug Monitoring and Pharmacogenetics, Medical University of Gdańsk, Gdańsk, Poland
}

\section{Key Words}

Chronic kidney disease $\bullet$ Lipoprotein lipase $\bullet$ Triglycerides $\bullet$ Lipolysis $\bullet$ Lipoproteins $\bullet$ Very low density lipoprotein $•$ High density lipoprotein

\begin{abstract}
Background/Aims: Hypertriglyceridaemia (HTG) and reduction and dysfunction of high density lipoprotein (HDL) are common lipid disturbances in chronic kidney disease (CKD). HTG in CKD is caused mainly by the decreased efficiency of lipoprotein lipase (LPL)-mediated very low density lipoprotein triglyceride (VLDL-TG) lipolysis. It has not been clarified whether HDL dysfunction in CKD contributes directly to HTG development; thus, the aim of this study was to assess the impact of CKD progression on the ability of HDL to enhance LPL-mediated VLDLTG lipolysis efficiency. Methods: VLDL was isolated from non-dialysis patients in CKD stages 3 and 4 and from non-CKD patients. The VLDL was incubated with LPL at the constant LPL:VLDLTG ratio, in the absence or presence of HDL. After incubation, the VLDL was separated and the percentage (\%) of hydrolyzed TG was calculated. Results: HDL presence increased the lipolysis efficiency of VLDL isolated from CKD and non-CKD patients, for the VLDL-TG $>50$ $\mathrm{mg} / \mathrm{dl}$. Its effect was dependent on the VLDL-TG and HDL-cholesterol concentrations in the reaction mixtures: the higher the concentrations of VLDL-TG and HDL-cholesterol, the greater the effect. The positive impact of HDL on VLDL lipolysis was modified by CKD progression: the percentage of lipolyzed VLDL-TG in the presence of $\mathrm{HDL}$ decreased with a reduction in eGFR ( $r=0.43, p=0.009$ ), and for patients with stage 4 CKD, no positive impact of HDL on lipolysis was observed. The percentage of lipolyzed TG correlated negatively with apoE and apoCs content in VLDL, and positively with HDL-apoCII, as well as with VLDL and HDL apoCII/ apoCIII ratios. The progression of CKD was associated with unfavourable changes in VLDL
\end{abstract}

M. Cackowska and E. Wieczorek contributed equally to this work. 


\section{Kidney Blood Pressure Research}

and HDL composition; apoE and apoCs levels increased in VLDL with a decrease in eGFR whereas the HDL-cholesterol level decreased. Conclusion: The progression of CKD affects lipoprotein composition and properties, and modulates the positive impact of HDL on VLDL lipolysis efficiency. In CKD patients, HDL deficiency and dysfunction can directly affect hypertriglyceridaemia development.

\section{Introduction}

HTG is a major lipid abnormality in chronic kidney disease (CKD) and an important contributor to a marked increase of cardiovascular mortality in this group of patients [13]. HTG reflects an increased quantity of triglyceride-rich lipoproteins (TRL) in plasma, i.e. chylomicrons (CM) carrying dietary lipids, liver-derived very low density lipoproteins (VLDL), and/or their remnant particles. The main mechanisms of HTG development include the enhanced liver secretion and impaired plasma TRL clearance, among which the clearance pathway is considered to be the most important $[4,5]$.

Intravascular lipolysis mediated by lipoprotein lipase (LPL) is a key process in plasma TRL clearance. LPL hydrolyzes triglycerides (TG) into glycerol and free fatty acid (FFA). Due to TG hydrolysis, CM is converted to smaller CM remnants and VLDL - to intermediate density lipoprotein (IDL), which can be taken up by the liver via LDL receptors or it can be further lipolyzed, producing low density lipoprotein (LDL). TG hydrolysis is accompanied by the release from TRL the surface material containing phospholipids (PL), free cholesterol (FC), and exchangeable apolipoproteins (apo): apoE, apoCII and apoCIII, which are transferred to high density lipoprotein (HDL), affecting its maturation and plasma concentration $[4,6]$.

Reduced HDL concentration and disturbances of its composition are known atherosclerosis risk factors. However, the mechanisms by which HDL affects atherosclerosis development still remain a matter of debate [7]. Taking into consideration the close link between plasma metabolism of TRL and HDL, it raises the question if unfavorable changes of HDL do not affect TRL clearance and HTG development in CKD patients.

HTG in CKD is accompanied by a significant reduction in plasma HDL, disturbed HDL composition, and HDL dysfunction [8, 9]. HDL in CKD have reduced ability to promote cholesterol efflux from macrophages as well as HDL anti-oxidative, anti-inflammatory and vasoprotective properties are impaired $[8,10]$. Lipid and lipoprotein disturbances develop in the early stages of the disease and exaggerate with the progression of renal failure. Thus, patients with different stages of CKD display different severity of HTG and other lipid-related disturbances [11-13].

The aim of this study was to assess the impact of CKD progression on the ability of HDL to enhance LPL-mediated VLDL-TG lipolysis efficiency. We found that HDL enhanced lipolysis efficiency in a VLDL-TG and HDL concentration-dependent manner, but its positive effect was reduced with the progression of CKD.

\section{Materials and Methods}

\section{Study group}

Adult non-dialysis patients in CKD stages 3 and 4, being under the care of the Outpatient Clinic of the Nephrology Department of the Medical University of Gdansk, and non-CKD patients were recruited into the study (Table 1). The study exclusion criteria were: acute diseases within 3 months before the study, liver diseases, malignancy, diabetes, treatment with immunosuppressive agents, fibrates or heparin. 55\% of CKD patients and none of non-CKD patients received statins.

The serum lipid and apolipoprotein concentrations for CKD and non-CKD patients are presented in Table 1. $29 \%$ of CKD patients were normolipidemic (TC $<190 \mathrm{mg} / \mathrm{dl}, \mathrm{TG}<150 \mathrm{mg} / \mathrm{dl}$ ). $50 \%$ had hypercholesterolemia, 5\% - hypertriglyceridemia, and 16\% - mixed hyperlipidemia. 13 non-CKD patients 


\section{Kidney \\ Blood Pressure Research}

were normolipidemic, whereas 4 patients had TC values above $190 \mathrm{mg} / \mathrm{dl}$ (up to $206 \mathrm{mg} / \mathrm{dl}$ ).

All subjects gave a written informed consent for participation in the study. The study was approved by the Independent Bioethics Commission for Research of the Medical University of Gdansk (Poland).

\section{VLDL and HDL isolation}

Blood was drawn from the vein after overnight fasting into commercially available test tubes to obtain the serum. VLDL was isolated by ultracentrifugation [14]. HDL was isolated from infranatant after VLDL separation by combined precipitation-ultracentrifugation procedure [15]. Next, the VLDL and HDL were dialyzed (18 h, $4 \stackrel{\circ}{\circ}$ ) against $0.01 \mathrm{M}$ Tris-HCl buffer, $\mathrm{pH} 7.4$, containing $0.195 \mathrm{M}$ $\mathrm{NaCl}$ and $0.01 \% \mathrm{NaN}_{3}$.

\section{VLDL lipolysis}

VLDL was incubated with LPL isolated from bovine milk (Sigma Aldrich, St Louis, MI, USA) for $1 \mathrm{~h}, 37{ }^{\circ} \mathrm{C}$ at the constant LPL:VLDL-TG $4.8 \mu \mathrm{g} / \mathrm{ml}: 90 \mathrm{mg} / \mathrm{dl}$ ratio, in the absence or presence of HDL. The basic VLDLcholesterol:HDL-cholesterol (VLDL-C:HDL-C) weight ratio was 1:1. In some experiments the different amount of HDL, i.e. VLDL-C:HDL-C ratios 1:0.1, 1:0.25, 1:0.5, 1:0.75, 1:1 were used. Albumin (Sigma Aldrich) was added to the reaction mixtures as an FFA acceptor (final concentration $2 \%, \mathrm{w} / \mathrm{w})$. A respective volume of $0.01 \mathrm{M}$ Tris- $\mathrm{HCl}$ buffer, pH 7.4, containing $0.195 \mathrm{M} \mathrm{NaCl}$ and $0.01 \% \mathrm{NaN}_{3}$, corresponding to the volume of HDL and/or LPL, was added to align the differences in volumes between the reaction mixtures. After incubation, the VLDL was separated by immunoprecipitation with anti-apoB antibodies [16], and the concentration and the percentage of TG released from the VLDL during lipolysis was calculated.

\section{Biochemical analysis and electrophoresis}

The concentrations of lipids (total cholesterol (TC), HDL-C, TG, phospholipids (PL)) were measured using commercially available enzymatic kits obtained from Pointe Scientific and Wako Diagnostics. LDL-C was calculated using the Friedewald formula. Apolipoproteins B, E, CII and CIII were measured by immunonephelometry using kits obtained from Siemens Healthcare and Randox. Pre- $\beta 1$-HDL was determined using enzyme-linked immunoassay kit obtained from Sekisui Diagnostics. Serum creatinine was measured using the enzymatic method (Abbott Diagnostics). Agarose electrophoresis was performed using Hydragel 15 Lipoprotein(E) kit obtained from Sebia. Densitometric analysis of electropherograms was performed with GelScan V. 1.45 (K. Kucharczyk Electrophoretic Techniques, Poland).

\section{Statistical analysis}

Statistical analysis was performed using GraphPad Prism 4.03. Normality was assessed with the Kolmogorov-Smirnov test. Data was expressed as mean \pm standard deviation (mean \pm SD) or median and $25^{\text {th }}$ $75^{\text {th }}$ percentiles. Statistical significance between the two groups was determined by non-paired or paired $t$ test, between more than two groups - by ANOVA with Tukey post-hoc test. Values of $p<0.05$ were considered statistically significant.
Table 1. Characteristics of study groups. $\left(25^{\text {th }}-75^{\text {th }}\right.$ percentiles $)$

\begin{tabular}{|c|c|}
\hline Parameter & Value \\
\hline \multicolumn{2}{|l|}{ CKD patients } \\
\hline Number & 36 \\
\hline Age (years) & $68(61-74)$ \\
\hline Gender (patients numer) & Female (16), Male (20) \\
\hline CKD stage (patients number) & 3a (11), 3b (15), 4 \\
\hline eGFR-MDRD [ml/min/1.73m²] & $37 \pm 12$ \\
\hline Serum creatinine $[\mathrm{mg} / \mathrm{dl}]$ & $1.56(1.37-2.12)$ \\
\hline \multicolumn{2}{|l|}{ Lipids: } \\
\hline TC $[\mathrm{mg} / \mathrm{dl}]$ & $211 \pm 46$ \\
\hline $\mathrm{LDL}-\mathrm{C}[\mathrm{mg} / \mathrm{dl}]$ & $135 \pm 44$ \\
\hline HDL-C [mg/dl] & $51 \pm 14$ \\
\hline TG [mg/dl] & $119 \pm 51$ \\
\hline PL [mg/dl] & $215 \pm 32$ \\
\hline \multicolumn{2}{|l|}{ Apolipoproteins: } \\
\hline apoB [mg/dl] & $99 \pm 25$ \\
\hline apoE $[\mathrm{mg} / \mathrm{dl}]$ & $4.23(3.44-5.07)$ \\
\hline apoCII [mg/dl] & $3.35 \pm 0.86$ \\
\hline apoCIII [mg/dl] & $11.4 \pm 3.11$ \\
\hline \multicolumn{2}{|l|}{ Non-CKD patients } \\
\hline Number & 17 \\
\hline Age (years) & $24(23-26)$ \\
\hline Gender (patients number) & Female (11), Male (6) \\
\hline eGFR-MDRD & $\geq 60$ \\
\hline Serum creatinine [mg/dl] & $0.76 \pm 0.12$ \\
\hline \multicolumn{2}{|l|}{ Lipids: } \\
\hline $\mathrm{TC}[\mathrm{mg} / \mathrm{dl}]$ & $171 \pm 23$ \\
\hline $\mathrm{LDL}-\mathrm{C}[\mathrm{mg} / \mathrm{dl}]$ & $94 \pm 18$ \\
\hline HDL-C [mg/dl] & $63 \pm 15$ \\
\hline $\mathrm{TG}[\mathrm{mg} / \mathrm{dl}]$ & $74 \pm 30$ \\
\hline $\mathrm{PL}[\mathrm{mg} / \mathrm{dl}]$ & $208 \pm 27$ \\
\hline \multicolumn{2}{|l|}{ Apolipoproteins: } \\
\hline apoB [mg/dl] & $76 \pm 14$ \\
\hline apoE [mg/dl] & $4.2 \pm 1.0$ \\
\hline apoCII [mg/dl] & $2.73 \pm 0.74$ \\
\hline apoCIII [mg/dl] & $8.36 \pm 2.42$ \\
\hline
\end{tabular}




\section{Kidney Blood Pressure Research}

\section{Results}

For CKD patients, LPL-mediated lipolysis of VLDL-TG occurred in a VLDL-TG concentration and HDL presence dependent manners (Fig. 1). In the absence of HDL, for the VLDL-TG concentration below $\sim 50 \mathrm{mg} / \mathrm{dl}$, the concentration of lipolyzed TG increased proportionally with the concentration of VLDL-TG. For the VLDL-TG above $\sim 50 \mathrm{mg} / \mathrm{dl}$, the concentration of lipolyzed TG did not increase and reached a plateau on average of 53.2 \pm 5.2 $\mathrm{mg} / \mathrm{dl}$. Conversely, in the HDL presence the concentration of lipolyzed TG increased with the VLDL-TG, in the whole range of the examined VLDL-TG concentrations (Fig. 1A).

In the absence of HDL there was a strong negative correlation between the percentage of lipolyzed TG and the concentration of VLDL-TG in the reaction mixtures (Fig. 1B). For VLDLTG concentration above $\sim 50 \mathrm{mg} / \mathrm{dl}$, HDL presence significantly increased the percentage of hydrolyzed TG on average by $9.3 \%(p<0.0001)$, in comparison to mixtures without HDL. The higher the VLDL-TG concentration, the greater effect of the HDL on lipolysis efficiency was observed. For the VLDL-TG concentration $\sim 90 \mathrm{mg} / \mathrm{dl}$, HDL increased the percentage of lipolyzed VLDL-TG by 15\% (Fig. 1B).

A similar relationship between VLDL-TG concentration, HDL presence, and VLDL-TG lipolysis efficiency has been observed for non-CKD patients (Fig. 1, insert graphs).

The effect of HDL on VLDL-TG lipolysis efficiency was HDL concentration-dependent. For the reaction mixtures with an increasing amount of HDL, the percentage of lipolyzed VLDL-TG gradually increased - the means were $67.2 \%, 71.5 \%, 74.0 \%, 78.3 \%, 83.3 \%$, and 85.1\% for VLDL-C:HDL-C 1:0, 1:0.1, 1:0.25, 1:0.5, 1:0.75, 1:1 ratios, respectively (Fig. 2A).

The presence and concentration of HDL had influence on the electrophoretic mobility of VLDL remnants produced during lipolysis (Fig. 2B). The remnants displayed lower electrophoretic mobility than the VLDL-control. The remnants produced in the absence of HDL had the mobility between beta and pre-beta. The remnants produced in the presence of HDL had lower mobility than the remnants produced in the absence of HDL, and for the

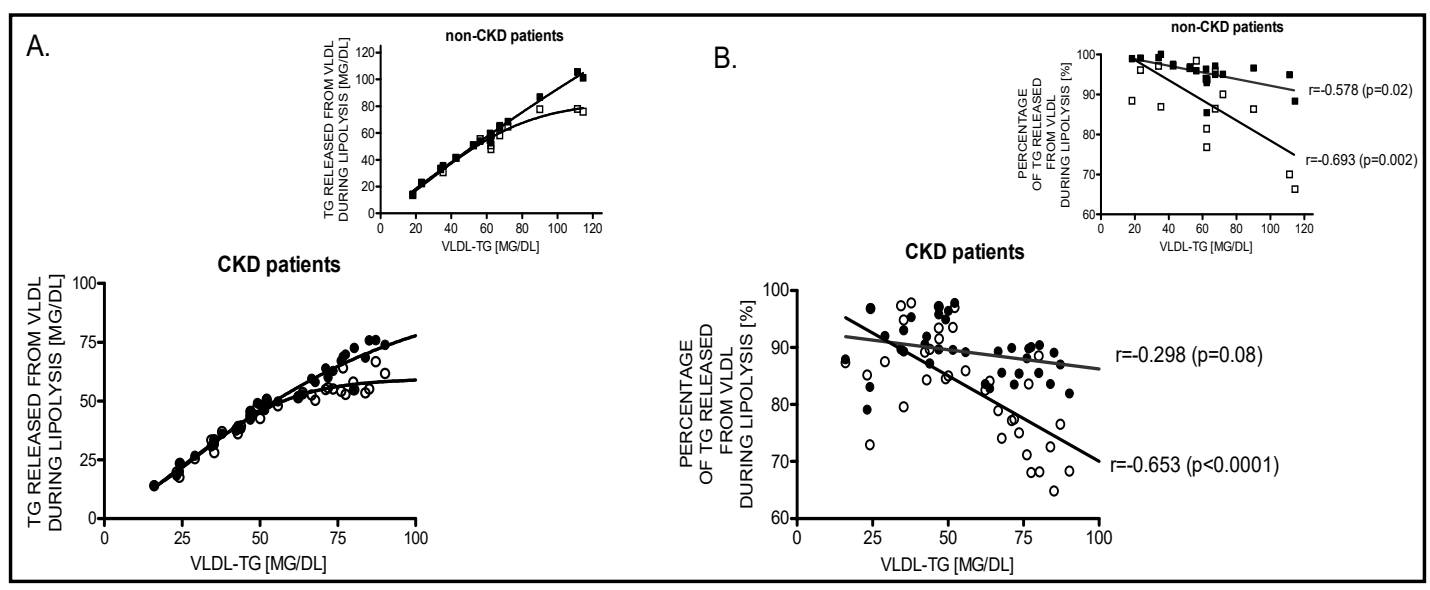

Fig. 1. Impact of HDL presence on VLDL-TG lipolysis efficiency for CKD patients (main graphs), and nonCKD patients (insert graphs). A. The relationship between VLDL-TG concentration in the reaction mixture $[\mathrm{mg} / \mathrm{dl}]$ and the concentration $[\mathrm{mg} / \mathrm{dl}]$ of lipolyzed TG, in the absence and presence of HDL (VLDL-C:HDL-C 1:1 weight ratio). B. The correlation between the VLDL-TG concentration in the reaction mixture [mg/ $\mathrm{dl}$ ] and the percentage [\%] of lipolyzed VLDL-TG, in the absence and presence of HDL (VLDL-C:HDL-C 1:1 weight ratio). Open circle/square - lipolysis in the absence of HDL, filled circle/square - lipolysis in the presence of HDL; $n=36$ (CKD), $n=17$ (non-CKD) patients. VLDL isolated from CKD and non-CKD patients was incubated with LPL (LPL:VLDL-TG $4.8 \mu \mathrm{g} / \mathrm{ml}: 90 \mathrm{mg} / \mathrm{dl}, 1$ hour, $37^{\circ} \mathrm{C}$ ), in the absence and presence of HDL. After incubation the VLDL was separated by immunoprecipitation, the TG was measured and the amount of lipolyzed TG was calculated. 


\section{Kidney \\ Blood Pressure Research}

remnants produced in the presence of the highest amount of HDL (VLDL-C:HDL-C 1:1 weight ratio), the electrophoretic mobility was the lowest (Fig. 2B).

The effect of HDL on VLDL-TG lipolysis efficiency was modified by CKD (Fig. 3). The percentage of lipolyzed VLDL-TG in the presence of HDL was the highest for non-CKD patients, reaching, on average, 95\%. For CKD patients, the lipolysis efficiency was lower than for non-CKD patients (mean 89\%, p<0.0001) and decreased with a decrease of eGFR (Fig. 3). In comparison to non-CKD patients and CKD patients with stage 3a, for patients with stage 4 CKD the \% of lipolyzed VLDL-TG in the presence of HDL was significantly lower, on average of $9 \%$ and $6 \%$, respectively (Fig. 3B). Moreover, for patients with stage 4, HDL did not increased significantly the \% of lipolyzed VLDL-TG, as it was observed for non-CKD patients and CKD patients with stage 3a and 3b (Fig. 3B). In the absence of HDL, the lipolysis efficiency for non-CKD patients was slightly higher than for CKD patients, but there was not statistically significant difference between the groups (Fig. 3B, $p=0.2$ ).

The effect of HDL presence on VLDL-TG lipolysis efficiency was not affected by statin treatment. The mean percentages of lipolyzed VLDL-TG in the absence and presence of HDL was $83.3 \pm 11$ and $89.1 \pm 3.8(p<0.05$, HDL( -$)$ vs HDL $(+))$ for patients not receiving statins, and 83.4 \pm 9.0 and $89.3 \pm 5.4(p<0.01$, HDL $(-)$ vs HDL $(+))$ for patients receiving statins, respectively.

The percentage of lipolyzed VLDL-TG correlated negatively with VLDL-apoE $(r=-0.493$, $p=0.002)$, VLDL-apoCII ( $\mathrm{r}=-0.424, p=0.01)$, and VLDL-apoCIII $(\mathrm{r}=-0.441, p=0.007)$. Positive correlations were observed for HDL-apoCII $(\mathrm{r}=0.362, p=0.03)$ and VLDL and HDL apoCII/apoCIII ratios ( $\mathrm{r}=0.310, p=0.06$, and $\mathrm{r}=0.426, p=0.01$, respectively).

The eGFR level was associated with the changes in lipid and lipoprotein profile in CKD patients (Table 2). The concentration of serum TG and apoCIII as well as the concentration of VLDL compounds such as TG, PL, cholesterol, apoE and apoCs increased with a decrease of eGFR. Conversely, the level of HDL-C decreased, but the concentration of pre $\beta_{1}$-HDL increased with a decrease of eGFR (Table 2).

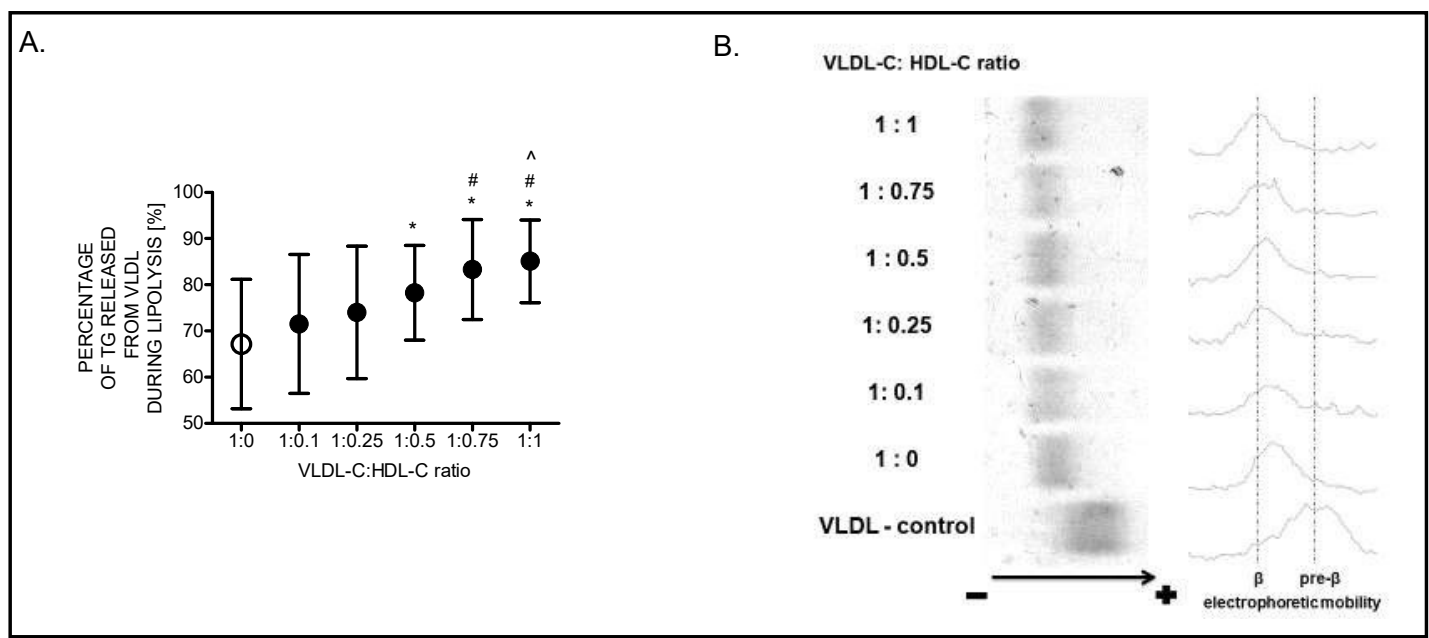

Fig. 2. Impact of HDL concentration on VLDL-TG lipolysis efficiency for CKD patients. A. The impact of different amount of HDL (VLDL-C:HDL-C 1:0, 1:0.1, 1:0.25, 1:0.5, 1:0.75, 1:1 weight ratios) on the percentage [\%] of lipolyzed VLDL-TG. Data are presented as mean \pm SD. *vs 1:0, "vs 1:0.1, ^ vs 1:0.25, p<0.05; $n=5$. B. Exemplary electropherogram of VLDL control and VLDL remnants obtained after LPL-mediated lipolysis in the absence or presence of a different amount of HDL. VLDL isolated from non-dialysis CKD patients was incubated with LPL (LPL:VLDL-TG $4.8 \mu \mathrm{g} / \mathrm{ml}: 90 \mathrm{mg} / \mathrm{dl}, 1$ hour, $37^{\circ} \mathrm{C}$ ), in the absence and presence of HDL. After incubation the VLDL was separated by immunoprecipitation, the TG was measured and the amount of lipolyzed TG was calculated. The reaction mixtures were separated by agarose gel electrophoresis and stained with Sudan black B. 


\section{Kidney Blood Pressure Research}

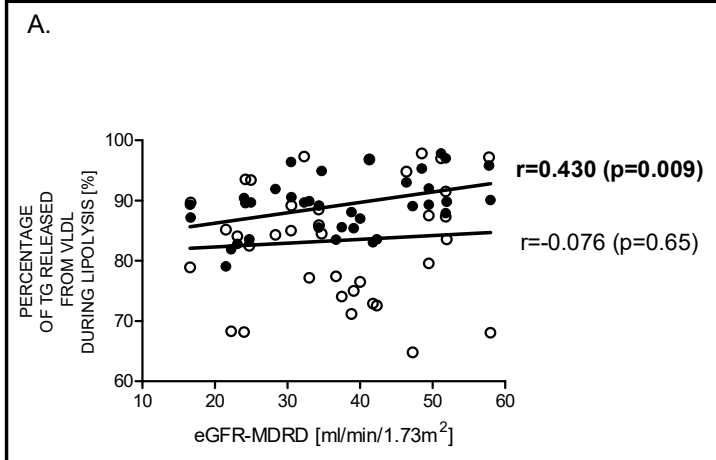

B.

Fig. 3. Impact of CKD progression on VLDL-TG lipolysis efficiency. A. The correlation between the eGFRMDRD [ml/min/1.73 $\mathrm{m}^{2}$ ] and the percentage [\%] of lipolyzed VLDL-TG, in the absence and presence of HDL. Open circle - lipolysis in the absence of HDL, filled circle - lipolysis in the presence of HDL; $n=36$. B. The percentage [\%] of lipolyzed VLDL-TG for non-CKD $(n=17)$ and CKD patients with stage $3 a(n=11), 3 b(n=15)$ and $4(\mathrm{n}=10) \mathrm{CKD}$, in the absence and presence of HDL. Data are presented as mean \pm SD; ${ }^{* * *}$ vs CKD stages $3 \mathrm{~b}$ and 4, p<0.001, *vs CKD stage 4, p<0.05, \#vs HDL (-), p<0.05. VLDL isolated from non-CKD and CKD patients was incubated with LPL (LPL:VLDL-TG $4.8 \mu \mathrm{g} / \mathrm{ml}: 90 \mathrm{mg} / \mathrm{dl}, 1$ hour, $37^{\circ} \mathrm{C}$ ) in the absence and presence of HDL (VLDL-C:HDL-C 1:1 weight ratio). After incubation the VLDL was separated by immunoprecipitation, the TG was measured and the amount of lipolyzed TG was calculated.

\section{Discussion}

In this study we show that the HDL presence and concentration significantly affects the VLDL lipolysis efficiency, and its positive effect is modified by the progression of CKD.

For both CKD and non-CKD patients, in the absence of HDL the percentage of lipolyzed VLDLTG ranged from $65 \%$ to $98 \%$ and was dependent on VLDL-TG concentration. HDL presence increased the VLDL-TG lipolysis efficiency in a VLDL-TG and HDLconcentration-dependent manners, and influenced the features of the generated remnants. The remnants produced in the presence of HDL had electrophoretic mobility corresponding to LDL $(\beta)$, whereas the remnants produced in the absence of HDL displayed higher mobility (between pre $\beta$ and $\beta$ ) corresponding to IDL, which can confirm delayed VLDL catabolism in the absence of HDL.

The high VLDL-TG concentration as a main reason of impaired lipolysis has been shown by others. Von Barlingen et al. found that VLDL isolated from HTG and normolipidemic subjects had the same $K_{\mathrm{m}}$ and $V_{\max }$ for LPL-mediated lipolysis reaction, despite the significant differences in VLDL lipid and protein composition, and they concluded that the delayed VLDL catabolism in HTG could be related to excessive VLDL-TG concentration [17]. The impact of basal TG concentration on lipolysis efficiency has also been confirmed in postprandial lipemia studies where the strong positive correlation between the magnitude of triglyceridemia in postprandial state and the fasting plasma TG concentration was observed $[18,19]$.

Table 2. Correlation coefficients for the relationship between the eGFRMDRD $\left[\mathrm{ml} / \mathrm{min} / 1.73 \mathrm{~m}^{2}\right]$ and lipid and apolipoprotein concentrations in CKD patients $(\mathrm{n}=36) .{ }^{*}$ Pearson's correlation coefficient, "Spearman's correlation coefficient

\begin{tabular}{lcc}
\hline Variable & Correlation coefficient $\mathrm{r}$ & $\mathrm{p}$ value \\
\hline TC in serum & $-0.165^{*}$ & 0.34 \\
LDL-C & $-0.181^{*}$ & 0.29 \\
VLDL-C & $-0.387^{\#}$ & 0.02 \\
HDL-C & $0.304^{*}$ & 0.07 \\
& & \\
TG in serum & $-0.324^{*}$ & 0.05 \\
VLDL-TG & $-0.300^{*}$ & 0.07 \\
HDL-TG & $-0.085^{*}$ & 0.62 \\
& & \\
PL in serum & $-0.067^{*}$ & 0.70 \\
VLDL-PL & $-0.310^{*}$ & 0.07 \\
HDL-PL & $0.282^{*}$ & 0.09 \\
& & \\
ApoB in serum & $-0.182^{*}$ & 0.29 \\
VLDL-apoB & $-0.246^{\#}$ & 0.15 \\
ApoE in serum & $0.271^{\#}$ & 0.11 \\
VLDL-apoE & $-0.430^{\#}$ & 0.009 \\
HDL-apoE & $0.367^{*}$ & 0.03 \\
& & \\
ApoCII in serum & $-0.081^{*}$ & 0.64 \\
VLDL-apoCII & $-0.307^{*}$ & 0.07 \\
HDL-apoCII & $0.081^{*}$ & 0.64 \\
ApoCIII in serum & $-0.419^{*}$ & 0.01 \\
VLDL-apoCIII & $-0.323^{\#}$ & 0.05 \\
HDL-apoCIII & $0.014^{*}$ & 0.9 \\
pre- $\beta_{1}$-HDL & $-0.502^{*}$ & 0.006 \\
\hline
\end{tabular}




\section{Kidney Blood Pressure Research}

Kidney Blood Press Res 2018;43:970-978

DOI: $10.1159 / 000490686$

Published onlıne: 18 June, 2018

(C) 2018 The Author(s). Published by S. Karger AG, Basel www.karger.com/kbr

Postprandial lipemia studies also confirm the observed by us the impact of HDL on VLDL lipolysis efficiency and TG serum level, since the magnitude of triglyceridemic response after fat meal intake correlated negatively with the HDL-C $[18,20]$.

The previous in vitro LPL-mediated VLDL lipolysis studies did not reveal the effect of HDL presence on lipolysis efficiency, but the beneficial effects of HDL on VLDL remnant features were shown. However, the impact of HDL on lipolysis efficiency has been observed for VLDL lipolysis mediated by other enzyme participating in the plasma VLDL lipolysis and its conversion to LDL, i.e. hepatic lipase (HL) $[21,22]$. HDL directly affected the rate of HLmediated VLDL lipolysis in an HDL concentration and composition-dependent manner. It was also shown that reconstituted HDL (rHDL) composed of apoAI and PL stimulated HLmediated VLDL lipolysis in a PL:apoAI ratio-dependent manner [22]. In our previous study, we showed that phosphatidylcholine liposomes increased the amount of surface material released from VLDL during LPL-mediated lipolysis and enhanced VLDL-TG lipolysis efficiency [23]. It can confirm that particles rich in PL, such as HDL, rHDL or liposomes, which are able to accept the surface material released from VLDL during lipolysis, are able to enhance VLDL lipolysis efficiency.

CKD patients display disturbances in lipoproteins composition [24], which could have an impact on TG LPL-mediated lipolysis susceptibility. However, the data concerning the influence of CKD on lipolysis efficiency is scarce and inconclusive. Lee et al. showed that the (VLDL+IDL) isolated from non-dialysis and dialysis CKD patients had a lower initial rate and total extent of lipolysis in comparison to lipoproteins isolated from the control subjects [25]. Arnadottir et al. observed that VLDL isolated from hemodialysis patients had the same initial reaction velocity, but it was lipolyzed to a lesser extent compared to VLDL isolated from healthy subjects [26]. In the postprandial lipemia studies performed by Weintraub et al. the postprandial triglyceride response in normolipidemic dialysis patients were similar to those in the normolipidemic nonrenal patients, whereas for hypertiglyceridemic subjects the triglyceridemic response were exaggerated and prolonged, independently of renal impairement [27]. On the other hand, Charlesworth et al. showed, that patients with renal diseases had a greater incremental rise in postprandial TG compared to control patients [28]. Saland et al. also found that the lipemic response was impaired in CKD children and the lower eGFR the greater postprandial lipemia occurred [29].

In our study, we observed that, for non-CKD patients, VLDL lipolysis efficiency in the presence of HDL was significantly higher than in CKD patients. Moreover, lipolysis efficiency decreased with a reduction in eGFR. For the patients with CKD stage 4, the presence of HDL did not increase the percentage of lipolyzed VLDL-TG. In the absence of HDL, lipolysis efficiency for non-CKD patients was slightly higher than for CKD patients, but the difference did not attain statistical significance. Thus it can be concluded that HDL disturbances in CKD play a very important role in delayed VLDL catabolism and HTG development.

The impact of HDL disturbances on VLDL lipolysis efficiency may be due to the increased amount of HDL subpopulation known as LPL inhibitor: preß-HDL [30] or due to changes in the interaction between VLDL and HDL caused by the modifications of their composition and properties in CKD [24]. Indeed, we observed that the lipolysis efficiency correlated negatively with apoE and apoCs content in VLDL, and positively with HDL-apoCII and VLDL and HDL apoCII/apoCIII ratios. Moreover, eGFR-MDRD correlated with the unfavourable changes in the lipid and apolipoprotein composition of VLDL and HDL. VLDL became richer in cholesterol and PL, as well as in apoE and apoCs. HDL-C was reduced, but the concentration of pre- $\beta_{1}-$ HDL increased with the progression of CKD. These results can confirm that CKD plays a crucial role in the VLDL catabolism, since pre- $\beta_{1}$-HDL as well as apoE and apoCIII are LPL inhibitors, and the reduction of HDL means the reduction of the number of acceptors of VLDL surface material that can also impair the lipolysis efficiency [23, 30, 31]. Thus it can be assumed that CKD affects HTG development through changes in lipoproteins concentration and composition, which has an impact on reducing the susceptibility of VLDL to lipolysis and/or HDL ability to interact with VLDL. 


\section{Kidney Bloód Pressure Research}

\section{Conclusion}

Our results prove that HDL directly increases the VLDL lipolysis efficiency in a VLDL-TG and HDL-C concentration-dependent manner, and its effect is modified by the progression of CKD. It can be concluded that a decrease of concentration and impairment of HDL function combined with an increase of TRL levels in CKD patients form a 'vicious circle' that can substantially impact one another, as well as the intensification of lipid disturbances and atherosclerosis development in this group of patients.

\section{Abbreviations}

apo (apolipoprotein); CKD (chronic kidney disease); CM (chylomicron); FC (free cholesterol); FFA (free fatty acid); HDL (high density lipoprotein); HL (hepatic lipase); HTG (hypertriglyceridaemia); LDL (low density lipoprotein); LPL (lipoprotein lipase); PL (phospholipid); TC (total cholesterol); TG (triglyceride); TRL (triglyceride-rich lipoprotein); VLDL (very low density lipoprotein)

\section{Acknowledgements}

This work was supported by the Medical University of Gdansk grants no. MN 010219/08/524, ST 02-0125/07/524 and ST 02-0004/07/122.

\section{Disclosure Statement}

We declare no conflict of interest.

\section{References}

1 Navaneethan SD, Schold JD, Arrigain S, Thomas G, Jolly SE, Poggio ED, Schreiber MJ, Jr, Sarnak MJ, Nally JV, Jr: Serum triglycerides and risk for death in Stage 3 and Stage 4 chronic kidney disease. Nephrol Dial Transplant 2012;27:3228-3234.

-2 Vaziri ND: Dyslipidemia of chronic renal failure: the nature, mechanisms, and potential consequences. Am J Physiol Renal Physiol 2006;290:F262-272.

-3 Krol E, Rutkowski B, Wroblewska M, Badzio T: Classification of lipid disorders in chronic hemodialyzed patients. Miner Electrolyte Metab 1996;22:13-15.

4 Bjornson E, Adiels M, Taskinen MR, Boren J: Kinetics of plasma triglycerides in abdominal obesity. Curr Opin Lipidol 2017;28:11-18.

-5 Chan DT, Dogra GK, Irish AB, Ooi EM, Barrett PH, Chan DC, Watts GF: Chronic kidney disease delays VLDLapoB-100 particle catabolism: potential role of apolipoprotein C-III. J Lipid Res 2009;50:2524-2531.

-6 Otarod JK, Goldberg IJ: Lipoprotein lipase and its role in regulation of plasma lipoproteins and cardiac risk. Curr Atheroscler Rep 2004;6:335-342.

7 Rye KA, Barter PJ: Cardioprotective functions of HDLs. J Lipid Res 2014;55:168-179.

8 Holzer M, Birner-Gruenberger R, Stojakovic T, El-Gamal D, Binder V, Wadsack C, Heinemann A, Marsche G: Uremia alters HDL composition and function. J Am Soc Nephrol 2011;22:1631-1641.

-9 Kon V, Yang H, Fazio S: Residual Cardiovascular Risk in Chronic Kidney Disease: Role of High-density Lipoprotein. Arch Med Res 2015;46:379-391.

10 Kaseda R, Jabs K, Hunley TE, Jones D, Bian A, Allen RM, Vickers KC, Yancey PG, Linton MF, Fazio S, Kon V: Dysfunctional high-density lipoproteins in children with chronic kidney disease. Metabolism 2015;64:263273. 


\section{Kidney \\ Blood Pressure Research}

11 Tsimihodimos V, Mitrogianni Z, Elisaf M: Dyslipidemia associated with chronic kidney disease. Open Cardiovasc Med J 2011;5:41-48.

-12 Rutkowski B, Łososowska R, Król E, Kisielnicka E, Zdrojewski Z, Szołkiewicz M, Niewęgłowski T, Chmielewski M, Sucajtys-Szulc E, Świerczyński J, Korczyńska J, Stelmańska E, Goyke E, Bogusławski W: Pathomechanism of hyperlipoproteinemia in chronic renal failure. Pol Merk Lek 2003;88:322-325.

13 Wróblewska M, Król E: Dyslipoproteinemias in kidney diseases. Pol Tyg Lek 1993;48:494-497.

14 McEneny J, Loughrey CM, McNamee PT, Trimble ER, Young IS: Susceptibility of VLDL to oxidation in patients on regular haemodialysis. Atherosclerosis 1997;129:215-220.

-15 Wroblewska M, Kortas-Stempak B, Szutowicz A, Badzio T: Phospholipids mediated conversion of HDLs generates specific apoA-II pre-beta mobility particles. J Lipid Res 2009;50:667-675.

-16 Cwiklinska A, Kortas-Stempak B, Gliwinska A, Pacanis A, Kuchta A, Wroblewska M: Interaction between VLDL and phosphatidylcholine liposomes generates new gamma-LpE-like particles. Lipids 2014;49:143153.

17 van Barlingen HH, Kock LA, de Man FH, Erkelens DW, de Bruin TW: In vitro lipolysis of human VLDL: effect of different VLDL compositions in normolipidemia, familial combined hyperlipidemia and familial hypertriglyceridemia. Atherosclerosis 1996;121:75-84.

18 Patsch JR, Karlin JB, Scott LW, Smith LC, Gotto AM, Jr: Inverse relationship between blood levels of high density lipoprotein subfraction 2 and magnitude of postprandial lipemia. Proc Natl Acad Sci U S A 1983;80:1449-1453.

19 O’Meara NM, Lewis GF, Cabana VG, Iverius PH, Getz GS, Polonsky KS: Role of basal triglyceride and high density lipoprotein in determination of postprandial lipid and lipoprotein responses. J Clin Endocrinol Metab 1992;75:465-471.

20 Couch SC, Isasi CR, Karmally W, Blaner WS, Starc TJ, Kaluski D, Deckelbaum RJ, Ginsberg HN, Shea S, Berglund L: Predictors of postprandial triacylglycerol response in children: the Columbia University Biomarkers Study. Am J Clin Nutr 2000;72:1119-1127.

-21 Ramsamy TA, Neville TA, Chauhan BM, Aggarwal D, Sparks DL: Apolipoprotein A-I regulates lipid hydrolysis by hepatic lipase. J Biol Chem 2000;275:33480-33486.

22 Ramsamy TA, Boucher J, Brown RJ, Yao Z, Sparks DL: HDL regulates the displacement of hepatic lipase from cell surface proteoglycans and the hydrolysis of VLDL triacylglycerol. J Lipid Res 2003;44:733-741.

23 Cwiklinska A, Gliwinska A, Senderowska Z, Kortas-Stempak B, Kuchta A, Dabkowski K, Jankowski M: Impact of phosphatidylcholine liposomes on the compositional changes of VLDL during lipoprotein lipase (LPL)mediated lipolysis. Chem Phys Lipids 2016;195:63-70.

24 Attman PO, Alaupovic P, Tavella M, Knight-Gibson C: Abnormal lipid and apolipoprotein composition of major lipoprotein density classes in patients with chronic renal failure. Nephrol Dial Transplant 1996;11:63-69.

-25 Lee DM, Knight-Gibson C, Samuelsson 0, Attman PO, Wang CS, Alaupovic P: Lipoprotein particle abnormalities and the impaired lipolysis in renal insufficiency. Kidney Int 2002;61:209-218.

-26 Arnadottir M, Dallongeville J, Fruchart JC, Nilsson-Ehle P: Very-low-density lipoprotein of uremic patients is a poor substrate for bovine lipoprotein lipase in vitro. Metabolism 1996;45:686-690.

-27 Weintraub M, Burstein A, Rassin T, Liron M, Ringel Y, Cabili S, Blum M, Peer G, Iaina A: Severe defect in clearing postprandial chylomicron remnants in dialysis patients. Kidney Int 1992;42:1247-1252.

-28 Charlesworth JA, Kriketos AD, Jones JE, Erlich JH, Campbell LV, Peake PW: Insulin resistance and postprandial triglyceride levels in primary renal disease. Metabolism 2005;54:821-828.

29 Saland JM, Satlin LM, Zalsos-Johnson J, Cremers S, Ginsberg HN: Impaired postprandial lipemic response in chronic kidney disease. Kidney Int 2016;90:172-180.

-30 Cheung AK, Parker CJ, Ren K, Iverius PH: Increased lipase inhibition in uremia: identification of pre-betaHDL as a major inhibitor in normal and uremic plasma. Kidney Int 1996;49:1360-1371.

-31 Yamamoto M, Morita SY, Kumon M, Kawabe M, Nishitsuji K, Saito H, Vertut-Doi A, Nakano M, Handa T: Effects of plasma apolipoproteins on lipoprotein lipase-mediated lipolysis of small and large lipid emulsions. Biochim Biophys Acta 2003;1632:31-39. 